\title{
An Algorithm for Computing Customized 3D Printed Implants with Curvature Constrained Channels for Enhancing Intracavitary Brachytherapy Radiation Delivery
}

\author{
Animesh Garg, Sachin Patil, Timmy Siauw, J. Adam M. Cunha, \\ I-Chow Hsu, Pieter Abbeel, Jean Pouliot, Ken Goldberg
}

\begin{abstract}
Brachytherapy is a widely-used treatment modality for cancer in many sites in the body. In brachytherapy, small radioactive sources are positioned proximal to cancerous tumors. An ongoing challenge is to accurately place sources on a set of dwell positions to sufficiently irradiate the tumors while limiting radiation damage to healthy organs and tissues. In current practice, standardized applicators with internal channels are inserted into body cavities to guide the sources. These standardized implants are one-size-fits-all and are prone to shifting inside the body, resulting in suboptimal dosages. We propose a new approach that builds on recent results in 3D printing and steerable needle motion planning to create customized implants containing customized curvature-constrained internal channels that fit securely, minimize air gaps, and precisely guide radioactive sources through printed channels. When compared with standardized implants, customized implants also have the potential to provide better coverage: more potential source dwell positions proximal to tumors. We present an algorithm for computing curvature-constrained channels based on rapidly-expanding randomized trees (RRT). We consider a prototypical case of $O B / G Y N$ cervical and vaginal cancer with three treatment options: standardized ring implant (current practice), customized implant with linear channels, and customized implant with curved channels. Results with a twoparameter coverage metric suggest that customized implants with curved channels can offer significant improvement over current practice.
\end{abstract}

\section{INTRODUCTION}

Automation science addresses the accuracy and quality of processes in a variety of applications from manufacturing to healthcare. Each year, over 500,000 cancer patients worldwide are treated with brachytherapy [1], a form of radiotherapy where needles or implants are temporarily inserted into the body to guide small radioactive sources close to tumors (brachys: Greek for proximal). Brachytherapy is widely used to treat cancer in a number of anatomical sties: interstitial locations such as prostate, pelvic sidewall, breast, liver, brain; and intracavitary locations such as nasal cavity, throat, tongue, rectum, cervix, and the vaginal canal [2].

Under the current practice of high dose rate brachytherapy (HDR-BT), a radioactive source is guided through hollow needles or catheters (interstitial) or through channels inside a standardized implant (applicator) that is inserted into a body cavity (intracavitary). The radioactive source is then pushed all the way through the needle or implant channel using an attached wire, and precisely withdrawn by an automated afterloader that causes the source to dwell for specified times at specified points along the needle or channel to deliver the

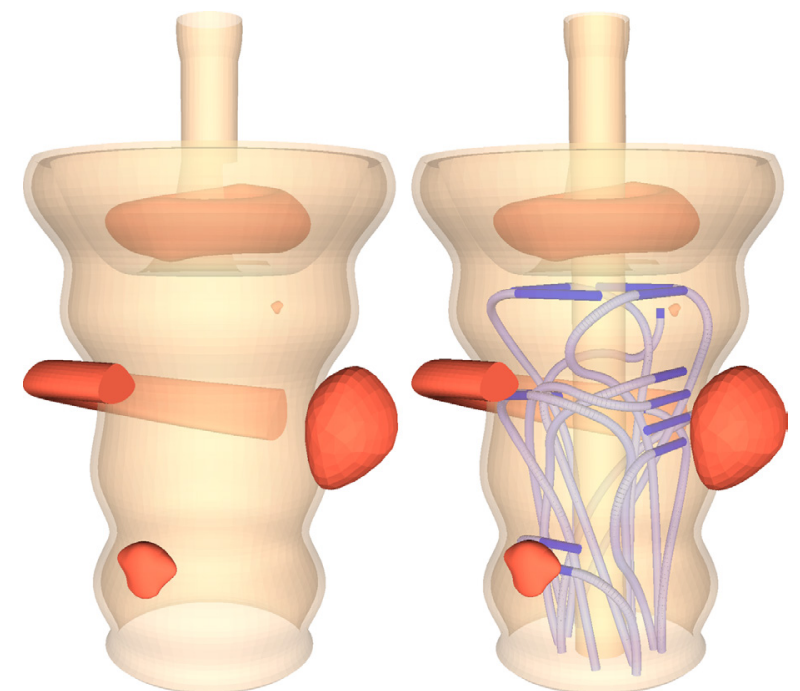

Fig. 1. Case study for OB/GYN cancer. Left: 3D model of customized implant for treating tumors of the cervix and endometrium of the vaginal cavity. The left figure shows an anatomical configuration of the vaginal canal (roughly cylindrical, transparent orange) with the cervix at the distal end (top of figure) and vaginal opening at the bottom of the figure. Five tumors, one around the cervix (top) and four on the vaginal sidewall, are depicted in solid red. Right: Customized implant with 12 curvature constrained channels (in light blue) generated by the algorithm. The small radioactive source (seed) can be precisely guided through each channel by a wire (controlled by a programmable afterloader) sequentially from each entry point (bottom) to each dwell segment (in solid blue) to precisely deliver treatment to the tumors.

desired radiation dose. Biological effectiveness requires the prescribed dose be divided into 2-4 iterations and delivered with intervening gaps of 5-6 hours. As illustrated in Figure 2, existing clinical methods employ standardized implants that do not conform to the patient anatomy allowing for relative movement, and only offer a fixed set of possible dwell position options for placing sources. In existing practice, patients are required to remain immobile over the course of treatment to maintain the geometric positions between anatomy and sources. Another limitation is that treatment quality depends on precisely positioning the sources to sufficiently irradiate the tumors while minimizing radiation delivered to healthy organs and tissues.

As noted by Magne et al. [3], "the proper placement of the applicator within vagina is the most important first step to avoid tumor underdosage or excessive dose to critical organs". We propose a new approach for HDR-BT intracavitary treatment that builds on recent results in $3 \mathrm{D}$ printing 


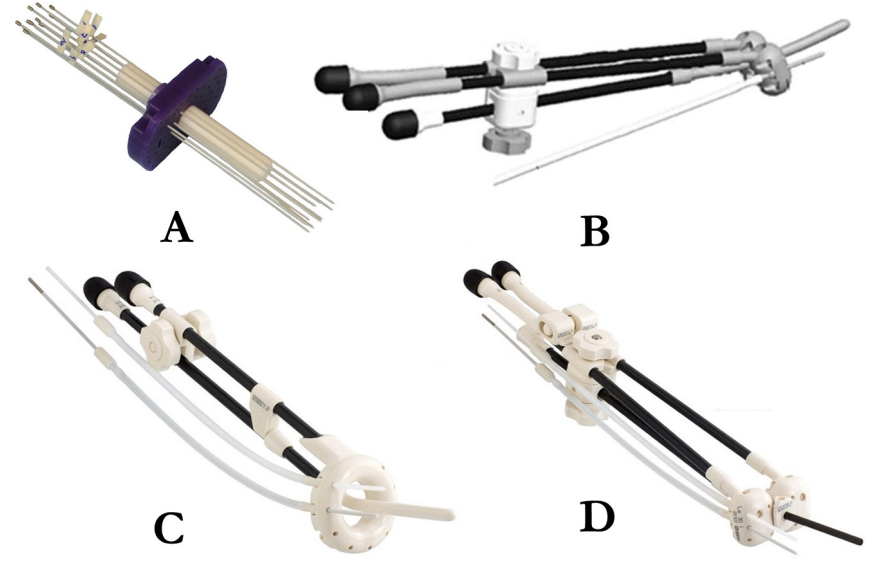

Fig. 2. Four standardized templates/applicators/implants commercially available for gynecological brachytherapy. (A) Vaginal cylinder applicator with 8 parallel catheters, (B \& D) Ovoids applicator with interstitial channels and uterine tandem applicator, (C) Ring applicator with interstitial channels and uterine tandem applicator. The uterine tandem applicator provides a channel for dwell positions along the uterine canal. The interstitial channels allow for applicator-guided insertion of catheters into the tissue surrounding the cervix

and steerable needle motion planning to design customized implants with interior curvature-constrained channels that can fit precisely and guide radioactive sources to customized dwell points proximal to cancerous tumors. Such curved channels have potential to reach targets that may not be reachable with existing methods. Customized implants can also provide a much better fit to increase patient comfort, reduce shifting due to movement and changes in bladder and bowel geometry, and permit patient mobility between treatment sessions.

We present an algorithm for computing curvature constrained channels that fit inside the specified implant geometry and meet dose and delivery requirements. The radiation source for HDR brachytherapy for treatment of GYN tumors is typically an ${ }^{192} \mathrm{Ir}$ core embedded in a steel capsule $0.9 \mathrm{~mm}$ in diameter and $\sim 5.0 \mathrm{~mm}$ in length [4] as shown in Figure 3. The cylindrical geometry imposes curvature constraints on the channels; given a channel diameter of $2.5 \mathrm{~mm}$, we calculate the minimum local curvature as $\sim 10 \mathrm{~mm}$.

Figure 1 illustrates an OB/GYN case study with typical cervical and vaginal tumors (the approach is also relevant to almost any other intracavitary HDR-BT). We next review related work. We define the problem in Section III and present the algorithm for computing curvature-constrained non-intersecting paths in Section IV. Section V describes the case study and results.

\section{RELATED WORK}

Automation science has been applied to a number of healthcare applications to improve quality of treatment by improving repeatability and reliability. Huang et al. [5] studied planning of robotic therapy and assessment of taskoriented functions for hand rehabilitation. Tervo et al. [6] and Solis et al. [7] explored the use of automation for studying human motor skills for medical task training. Mendez et al. [8] studied automatic control of anesthesia, and Subburaj

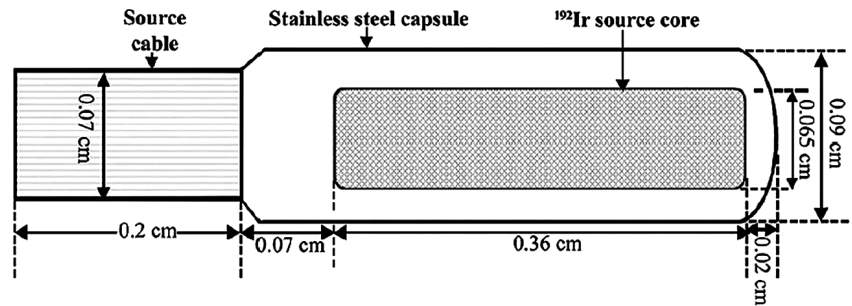

Fig. 3. Schematic of a typical ${ }^{192}$ Ir source used in GYN Brachythrapy [4](Permission pending).

et al. [9] studied computer assisted joint reconstruction surgery.

In our previous paper, Garg et al. [10], we addressed limitations imposed by standardized external templates for guiding linear needles for treatment of prostate cancer. We demonstrated how a set of linear brachytherapy needles could be accurately delivered in a non-parallel (skew-line) pattern by a specialized robot to avoid puncturing sensitive organs. In the present paper, we extend these ideas in several ways, considering how 3D printing can be used to achieve precise patient anatomy alignment without a robot and presenting an algorithm for computing curved interior channels through the 3D printed implant for delivering radioactive sources.

Potter et al. [11] [12] present recommendations on intracavity BT dose distributions for gynecological cancers. There are a number of commercially-available implants/applicators for treating cervical and endometrial cancers: Fletcher applicators [13], Utretch applicator [14], Vienna applicator [15] and Mold type applicators [3]. These standardized implants can be combined with linear catheters as illustrated in Figure 2. Used by many radiation oncologists, these intracavitary applicators include an intrauterine tandem and intravaginal ovoids, producing a pear-shaped dose distribution centered on the cervix, allowing a high dose to be delivered to the cervix while sparing bladder and rectum. Although these systems allow some adaptation to patient anatomy, patient movement (and filling of bladder and bowels) can cause shifts in the applicator position that result in undesired doses.

One exciting innovation is the approach described by Magne et al. [3], which proposes use of a customized implant created with a plaster vaginal impression that accurately shows the topography and extension of tumors and the specific anatomy of the vagina and cervix. In their experiments, two linear catheters and tandem shaft are inserted by the oncologist into the implant. The authors report decreased relative movement of implant while the patient is mobile over three days, thereby enabling less error between planned and delivered dose distributions. Treatment of patients with tumor extensions to the endometrial tissue of the vaginal wall often requires two separate implants if treated with standard applicators. A custom implant allows the oncologist to account for tumor extensions in a single iteration. The authors report their experience with more than 5000 patients and note that their method has three main advantages: personalized tailored treatment, MRI procedure compatibility without image quality disturbance, and increased patient comfort. We 
note that Magne et al prepare the mold implant manually and correct placement of catheters is highly dependent on oncologist's experience.

In the present paper we explore an extension where the plaster cast is scanned (or the patient anatomy segmented from MRI or CT scans) to create a precise 3D model that is provided as input to an algorithm for computing a set of internal curved channels that can be embedded into a plastic implant with 3D printing.

External templates for guiding linear needles for brachytherapy have also been studied. Roy et al. [16] explored the use of precision machining of linear needle paths. These templates and paths were not generated algorithmically.

Recent advances in 3D printing (also known as additive manufacturing) are poised to have major impact on many fields as described by Lipson [17] and Gershenfeld [18]. Jacobs [19] is an early introduction. Non-toxic, FDA approved materials are allowing 3D printed parts to be used for medical applications [20] such as bone replacement [21] and oral surgery implants [22].

A growing body of research has been reported on motion planning for steering needles [23] [24] [25]. The objective is to steer a flexible needle with curvature constraints through tissue to internal targets by exploiting asymmetries at the needle tip. Such needles can reach targets that cannot be reached by stiff linear needles. The needle is a nonholonomic system and is related to motion planning for fixed-wing aircraft [26] [27].

Computing a set of internal channels is a similar problem in that curvature is contrained but has the distinct advantage that there is no uncertainty due to tissue properties or needle mechanics: channels can be printed with extreme accuracy. It is also important that channels do not intersect. We build on prior work by Patil et al. [24] which uses rapidly exploring random trees (RRT) [28] for planning curvature constrained paths for steerable needles [25].

\section{Problem Definition}

The objective is to compute a set of non-intersecting curvature-constrained channels within the implant that reach targets proximal to tumors for delivery of radiation and if needed, a report of which tumor zones cannot be reached.

The input is the registered pre-operative geometry from a combination of 3D scan of the plaster cast and CT (or MRI) scan of the patient. This input includes: external geometry of the implant specified as a triangle mesh; the desired entry zone at the base of the implant for all channels; and the locations of tumors and organs-at-risk (OAR) (vaginal wall, cervix, rectum, urethra, bladder, uterus). The channel layout problem can then be stated as follows:

Objective: Given a 3D model of the implant volume $I$, which may include internal voids that will be treated as obstacles for channels, a set of 3D cancerous tumors that require radiation treatment $\mathcal{T}$, a specification of the entry region at the base of the implant $E$, the maximum allowable entry angle (deviation from normal) $\alpha$, the minimum radius of curvature of the channel, $r_{\min }$, and the channel diameter, $w$, corresponding to the width of the catheter carrying the source, the objective is to compute a set of non-intersecting curvature constrained channels $\mathcal{C}=\left\{C_{1}, C_{2}, \ldots, C_{N}\right\}$ starting from $E$ that lie within $I$ and are proximal to as much of the set $\mathcal{T}$ as possible.

\section{A. Coverage Quality Metric}

The ability to deliver radiation doses depends on the arrangement of potential source dwell points and their proximity to tumors. The radiation dosage at radius $r$ follows an inverse square law. We measure the quality of an implant by the percentage of tumor volume that is "covered" by the set of dwell points, where coverage is a function of coverage distance between a dwell point (source) and a tumor point (target). Higher quality reduces the the maximum dwell time needed to treat tumors and in turn the potential for hot spots that can harm healthy tissue. Alternate quality metrics can be based on inverse dose planning [4], which we will study in future work.

To compare implants and channels for a given set of tumors $\mathcal{T}$, we consider the set of reachable dwell positions and how thoroughly they "cover" the set of tumors. Consider a set of reachable dwell positions $\mathcal{S}$ (for instance in case of 3D printed implants these are evenly spaced inside reachable dwell segments). We discretize the set of tumors into a set of evenly spaced points $d T$. We quantify the proximity of a dwell position $d S$ from a tumor point $d T$ with the "coverage radius" $\delta$ such that: if $d S$ lies within a ball of radius $\delta$ centered at $d T$, then $d S$ is said to cover $d T$. It is also helpful to consider cases where tumor points can be covered by some multiple $n$ of dwell points. Hence the cover $\mathrm{C}$ of $d T$ is the set

$$
\mathrm{C}(d T, \delta)=\left\{d S:\|d S-d T\|_{2} \leq \delta, d S \in \mathcal{S}\right\}
$$

We define the quality of coverage $\mathcal{Q}(n, \delta)$ as the percentage of tumor volume such that each tumor point within that volume $d T \in \mathcal{T}^{\prime}, \mathcal{T}^{\prime} \subseteq \mathcal{T}$ is covered by at least $n$ dwell positions within a ball of radius $\delta$ centered at $d T$.

$$
\mathcal{Q}(n, \delta)=\frac{1}{|\mathcal{T}|} \int_{\mathcal{T}} I\{|C(d T, \delta)| \geq n\} d T .
$$

where $I\{\cdot\}$ is the indicator function and $|\cdot|$ is set cardinality. Reaching $100 \%$ coverage with smaller radiation radius and more dwell positions can reduce occurrence of hot spots and increase dose conformality to the tumor geometry to spare healthy tissue.

\section{Channel Layout Algorithm (CLA)}

The Channel Layout Algorithm (CLA) is summarized in Alg. 1. The first step is generating a set of dwell segments proximal to the given set of tumors. Starting from the dwell segment most distal to the entry zone, we use the curvature constraints to construct an RRT backward from the segment toward the entry zone, stopping if/when we find a channel that avoids obstacles. We then treat this channel as an obstacle and consider the next dwell segment until all dwell 


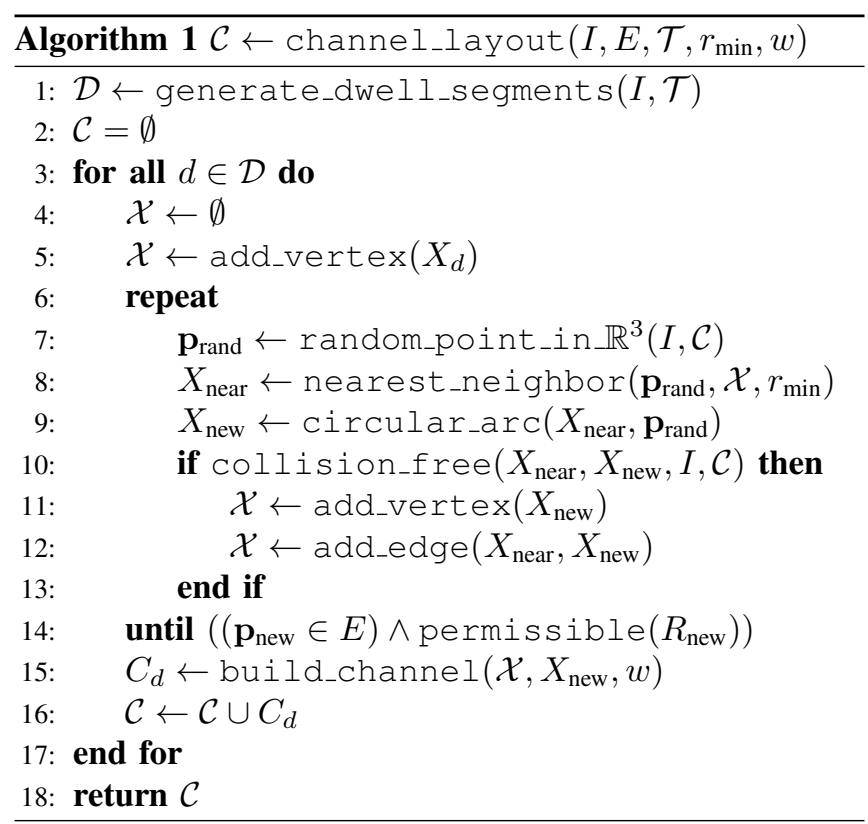

segments are considered. We describe each step in detail below.

generate_dwell_segments(.): We start by computing a candidate set of dwell segments, which are linear segments near tumors that may include multiple potential source dwell positions. We can also consider curved dwell segments and segments in alternate orientations.

Given the set of tumors $\mathcal{T}$ and the implant volume $I$, we compute the set of dwell segments $\mathcal{D}$ as follows. We discretize the implant volume with a regular voxel grid, where each voxel is a cube of side length equal to the channel width $w$. Since the surface of the implant volume is represented as a discretized triangular mesh, we mark all the triangles from which the outward facing surface normals intersect the tumor surfaces. Given the marked triangles, we project them in the direction of the inward facing surface normal by a distance $w$ to account for the channel width, and mark all voxels intersected by the projected triangles. These marked voxels represent a discretization of the volume that should ideally be covered with the dwell segments. This is also known as the "pencil packing problem," for which finding an optimal solution is NP-hard [29]. Currently, we suboptimally select a set linear segments that cover the marked voxels (see Section VI for planned extensions to this step).

For each dwell segment $\mathcal{D}$, we compute a channel inside the implant volume that reaches it or a report that no channel can be found. We consider the dwell segments in decreasing order of distance from the entry region $E$. The medial axis of each curvature constrained channel can be parameterized as a sequence of circular $\operatorname{arcs}\left\{\Psi_{1}, \Psi_{2}, \ldots, \Psi_{n}\right\}$ in 3D space, where each circular arc $\Psi_{i}$ is parameterized as a tuple $\left[l_{i}, \phi_{i}, r_{i}\right]^{T}$ (Figure 4). Here, $l_{i}$ is the length of the arc, $r_{i}>r_{\min }$ is the radius of the arc, and $\phi_{i}$ is the twist applied to the tangential frame at the end of $\Psi_{i}$ that rotates

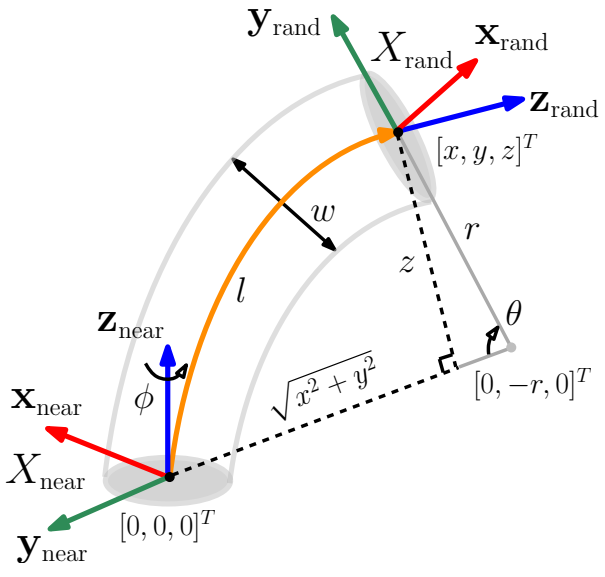

Fig. 4. The medial axis of each channel is parameterized with a sequence of circular arcs $\left\{\Psi_{1}, \Psi_{2}, \ldots, \Psi_{n}\right\}$. We show one such circular arc here (orange) parameterized as a tuple $[l, \phi, r]$. The channel is obtained by sweeping a disk of diameter $w$ along the length of the arc. This arc connects the state $X_{\text {near }} \in S E(3)$ at the nearest tree node to the randomly sampled point $\mathbf{p}_{\text {rand }} \in \mathbb{R}^{3}$. We assume that the medial axis of the channel is oriented along the local $\mathbf{z}$-axis at each point along the arc. The circular arc is constructed by rotating the local frame $X_{\text {near }}$ by an angle $\theta$ around a line parallel to the local $\mathbf{x}$-axis and passing through the point $[0,-r, 0]^{T}, r>$ $r_{\min }$. The rotation $\phi$ rotates the tangential frame at the end of one circular arc to align it with the plane that contains the subsequent circular arc.

the plane containing the $\operatorname{arc} \Psi_{i}$ to the plane that contains the arc $\Psi_{i+1}$. The channel is constructed by sweeping a circle of diameter $w$ along the medial axis.

Although the channels are constructed in 3D space, the state space of the layout problem comprises of both the 3D position and orientation $(S E(3))$ because of the constraints on the channel curvature. The position and orientation constraint at the end of each dwell segment $d \in D$ can be described as $X_{d}=\left[\begin{array}{cc}R_{d} & \mathbf{p}_{d} \\ \mathbf{0} & 1\end{array}\right] \in S E(3)$ comprising of the position $\mathbf{p}_{d}$ of the end of the segment and rotation matrix $R_{d}$ encoding the orientation of the dwell segment in 3D. Without loss of generality, we assume that the dwell segment $d$ is oriented along the $\mathbf{z}$-axis of the local coordinate frame attached to the end of dwell segment.

Recent results in motion planning for nonholonomic systems emphasize sampling-based methods such as the Rapidly-exploring Random Tree (RRT) planner [28] where the probability of finding a solution converges to one, if such a solution exists, as the number of samples approaches infinity. We employ this approach building on an algorithm to compute curvature constrained needle paths in 3D space [24]. Given a dwell segment $d \in \mathcal{D}$, we use the planner to compute the medial axis of the channel while staying within the implant volume and avoiding obstacles and the set of existing channels $\mathcal{C}$ in the environment. We plan backwards starting from the dwell segment $d$ to the entry region $E$ because the larger entry region is less constrained.

Given initial state $X_{d}$ and entry region, the algorithm incrementally builds a tree $\mathcal{X}$ over the state space, while conforming to nonholonomic motion constraints of the system and avoiding obstacles. As described in Patil et al. [24], building the tree in the $S E(3)$ state space directly is compu- 
tationally inefficient, so we sample a random point $\mathbf{p}_{\text {rand }} \in$ $\mathbb{R}^{3}$ rather than a random state $X_{\text {rand }} \in S E(3)$. The planner then identifies a node in the tree $X_{\text {near }}$ that is closest to the sample $\mathbf{p}_{\text {rand }}$, as defined by a specified distance metric $\rho[\cdot]$. The sample $\mathbf{p}_{\text {rand }}$ is then connected to $X_{\text {near }}$ using a circular arc parameterized by the tuple $[l, \phi, r]^{T}$. If the circular arc does not collide with the implant volume or existing channels and the minimum clearance from the obstacles is at least the channel width $w$, we add the arc as an edge in the tree. This process is repeated until either the tree $\mathcal{X}$ connects $X_{d}$ and $E$ or the available computation time is exceeded, in which case the planner reports that a solution cannot be found. The medial axis of the channel can then be extracted from the tree by traversing backwards from the entry region to the dwell segment that corresponds to the root of the tree.

random_point_in_ $\mathbb{R}^{3}(\cdot)$ : We sample a random point $\mathbf{p}_{\text {rand }} \in \mathbb{R}^{3}$ within the implant volume $I$ that is not collision with any of the channels in $\mathcal{C}$. The sampled point can then be connected to a given state $X_{\text {near }}=\left[\begin{array}{cc}R_{\text {near }} & \mathbf{p}_{\text {near }} \\ 0 & 1\end{array}\right]$ directly using a circular arc parameterized by $[l, \phi, r]^{T}$, where $l$ is the arc length, $\phi$ is the change in orientation of the node $X_{\text {near }}$

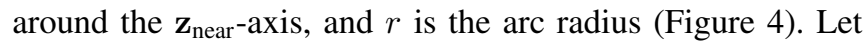
$[x, y, z]^{T}=R_{\text {near }}^{T}\left(\mathbf{p}_{\text {rand }}-\mathbf{p}_{\text {near }}\right)$ be the coordinates of $\mathbf{p}_{\text {rand }}$ in the local coordinate frame of $X_{\text {near. }}$ The parameters of the circular arc are then given by:

$$
\begin{aligned}
r & =\frac{x^{2}+y^{2}+z^{2}}{2 \sqrt{x^{2}+y^{2}}} \\
\phi & =\arctan (x,-y) \\
l & =r \arctan \left(z, r-\sqrt{x^{2}+y^{2}}\right) .
\end{aligned}
$$

To build toward the entry zone, we incorporate two forms of biasing when constructing the tree. First, sample from the entry zone with a higher probability than the rest of the implant volume. Second, whenever a new node $X_{\text {new }}$ is added to the tree, the planner attempts to connect $X_{\text {new }}$ to a randomly sampled point in the entry zone $E$.

nearest_neighbor (.): We use the distance measure proposed by Patil et al. [24] that is customized for nonholonomic systems with curvature constraints to select the tree node that is nearest to the sampled point $\mathbf{p}_{\text {rand }}$. Since the channel has a minimum radius of curvature $r_{\min }$, not all sampled points will be reachable from a given state. The reachable set from a state $X_{\text {near }}=\left[\begin{array}{cc}R_{\text {near }} & \mathbf{p}_{\text {near }} \\ 0 & 1\end{array}\right]$ consists of all points that can be connected to $\mathbf{p}_{\text {near }}$ by a circular arc that has a radius $r \geq r_{\min }$ and is tangent to the $\mathbf{z}_{\text {near-axis }}$ of the local coordinate frame. We use this definition of the reachable set to define the distance metric $\rho\left[X_{\text {rand }}, \mathbf{p}_{\text {rand }}\right]$ as the length of such a circular arc connecting $\mathbf{p}_{\text {rand }}$ and $X_{\text {near }}$ if $\mathbf{p}_{\text {rand }}$ is in the reachable set of $X_{\text {near }}$, and infinity otherwise.

$$
\boldsymbol{\rho}\left[X_{\text {rand }}, \mathbf{p}_{\text {rand }}\right]=\left\{\begin{array}{cl}
l(\equiv r \theta) & \text { if } r \geq r_{\min } \wedge \theta \geq 0 \\
\infty & \text { otherwise }
\end{array} .\right.
$$

circular_arc(.): Given a circular arc parameterized as $[l, \phi, r]^{T}$ and a maximum step size $\Delta$ to progress at each iteration of the RRT algorithm, we compute the position and orientation of the new node $X_{\text {new }}$ by composing a rotation

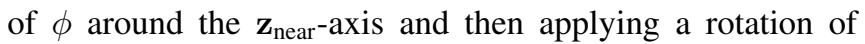
$\theta=\min \{l, \Delta\} / r$ around a line parallel to the $\mathbf{x}_{\text {near }}$-axis and passing through the point $[0,-r, 0]^{T}, r>r_{\min }$ in the local coordinate frame of $X_{\text {near. }}$.

collision_free $(\cdot)$ : To enable obstacle avoidance, only collision free arcs are added to the tree. We check if the circular arc connecting $X_{\text {near }}$ and $X_{\text {new }}$ is collision free by approximating it as a sequence of line segments and checking if all the segments are collision free. Since the obstacle definitions are obtained from segmentation of 3D scans, the obstacle meshes are likely to be non-manifold. We use the SOLID library [30] for detecting collisions with arbitrary, polyhedral obstacles at interactive rates. We also check if the minimum clearance of the circular arc is at least the channel width $w$ from the implant volume and existing channels to ensure that the channel that is constructed around the medial axis of this arc is collision free.

permissible $(\cdot)$ : Since the catheter carrying the source is inserted through the channels, we want the channel orientation at the entry region $E$ to as close as possible to perpendicular to $E$. We allow a cone of permissible orientations, i.e., the dot product of the local $\mathbf{z}$-axis at a point on the channel medial axis at the entry region and the normal to the entry region should be less than the maximum allowable entry angle (deviation from normal), $\alpha$.

build_channel(.): A channel is found when the position $\mathbf{p}_{\text {new }}$ of a newly added state $X_{\text {new }}$ is found to lie in the entry region $E$ and the orientation $R_{\text {new }}$ is permissible. By traversing the tree $\mathcal{X}$ backwards from $X_{\text {new }}$ to the root $X_{d}$, we obtain a path composed of piecewise circular arcs $\left\{\Psi_{1}, \Psi_{2}, \ldots, \Psi_{n}\right\}$ constituting the medial axis of the channel, each with radius $r>r_{\text {min }}$. We build the channel by sweeping a circle of diameter $w$ along the medial axis.

The channel is then added to the list of existing channels $\mathcal{C}$ and the process is repeated for the next most distant dwell segment until all dwell segments $\mathcal{D}$ are considered. As it may not be possible to find solutions for all dwell segments, we report a segment as unreachable if a maximum number of iterations of the RRT algorithm are exceeded and no valid path is found to the entry region $E$. It may be possible that alternate orderings of dwell segments could produce better coverange and we will consider other heuristics in future work.

\section{Case Study and Evaluation}

As a case study, we consider a 3D model of OB/GYN anatomy with comparable scale and relative sizes of tumors and organs based on Barnhart et al. [31]. For this example, the diameter of the cavity near cervix is $50 \mathrm{~mm}$ and diameter at the vaginal introitus is $28 \mathrm{~mm}$.

We consider three treatment methods: standardized ring implant (current practice), customized 3D Printed implant with linear channels, and customized 3D Printed implant with curved channels. We compare them with the coverage quality metric defined in Section III.

We first consider the standardized ring implant. The left image in Figure 5 shows a ring implant placed in the 


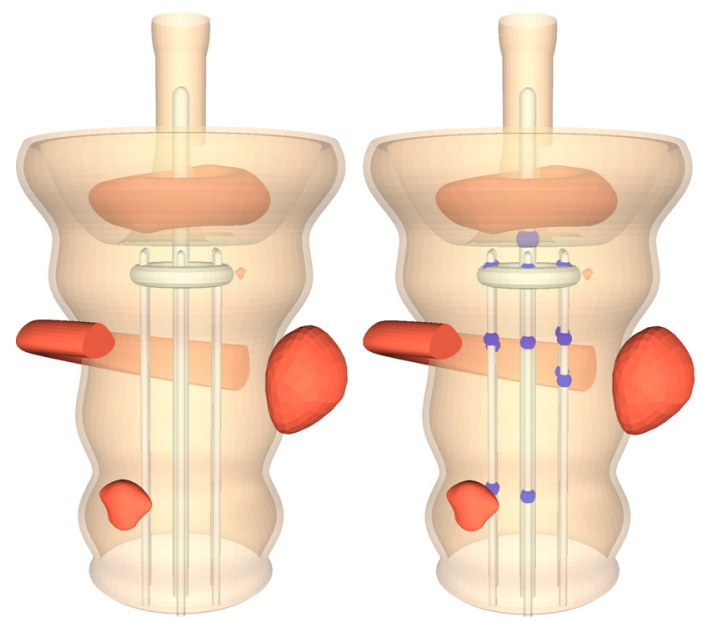

Fig. 5. Standardized ring implant (white) that cannot conform to patient anatomy. Only 18 dwell positions are reachable (in blue).

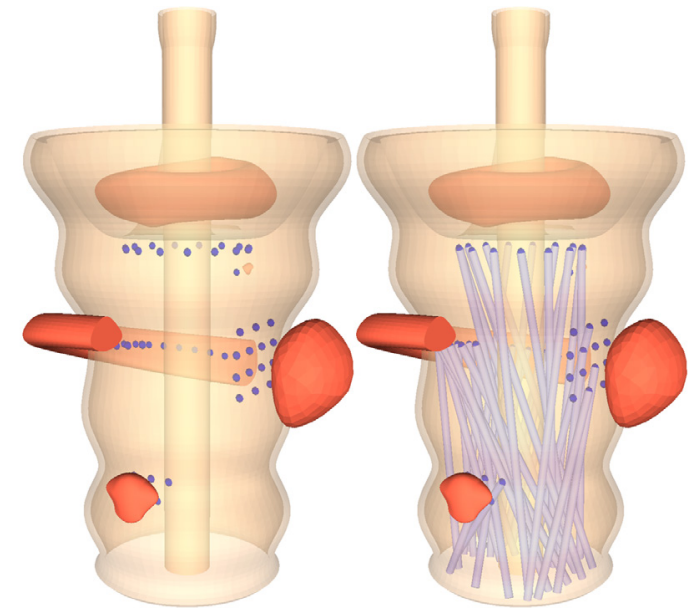

Fig. 6. 3D Printed implant with only linear channels: Left: 40 reachable dwell positions and segments. Right: achievable linear channels.

vaginal cavity. The ring implant contains a toroidal channel running around the interior of the ring and number (usually 6) of parallel catheter channels running parallel to the axis of symmetry of the ring along near its outer diameter. A central tube (uterine tandem) passes into the uterine canal via the cervix. In a clinical procedure, the ring implant is inserted by the physician and then the patient is scanned using either MR or CT imaging. After scanning, a physician digitally segments the anatomical structures and digitizes the positions of the catheters. Using these structures and the set of catheter positions defined by their geometry, dose optimization software determines the best subset of dwell positions and times at each of these positions. The right image in Figure 5 shows one such configuration of dwell positions superimposed on the implant.

Next we consider an alternative related to the plaster implant proposed by Magne et al. [3], where the channels are manually created by the clinician by pushing linear catheters into the soft material. The right image in the Figure 6 shows a set of linear channels (skew lines) that reach as many of the dwell positions as permitted by the size of the entry zone.

Finally we consider the implant with curvature-constrained

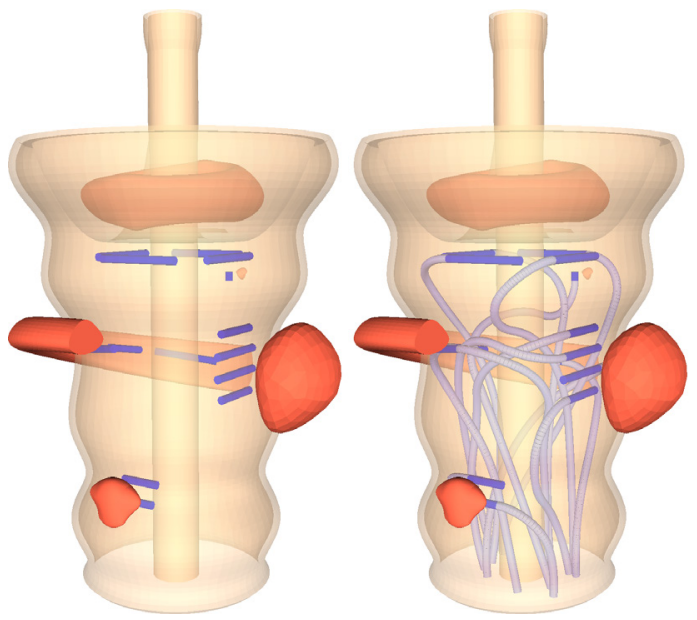

Fig. 7. 3D Printed implant with Curved Channels computed by the CLA algorithm: Left: 149 reachable dwell positions and segments. Right: channels computed by the CLA algorithm.

non-linear channels generated by the CLA algorithm: Figure 7.

The standardized ring implant can reach 18 potential radiation source dwell points, the 3D Printed implant with linear channels can reach 40 dwell points and the 3D Printed implant with curved channels can reach 149 dwell points. Table I lists the values of $\delta$ in $\mathrm{mm}$ at which coverage quality $\mathcal{Q}$ reaches $100 \%$. Figure 8 plots the quality metric for the three implant options (A),(B) and (C) as functions of coverage radius $\delta$ for $1,5,10$, and 15 dwell points respectively.

\section{Discussion AND Future Work}

At CASE 2012, we proposed a new approach to interstitial brachytherapy using a robot to precisely align linear needles Garg et al. [10]. In the present paper,o we propose a new approach to treating intracavitary brachytherapy using 3D printing and present an algorithm for generating curvatureconstrained internal non-linear channels. We consider a case-study with an OB/GYN cervical and vaginal cancer to compare three treatment options: standardized implant (current practice), customized implant with linear channels, and customized implant with curved channels. Results with a two-parameter coverage metric, summarized in Section V and Table 1, suggest that customized implants with curved channels can offer significant improvement over current

\begin{tabular}{|l||l|l|l|}
\hline \multicolumn{1}{|c||}{} & \multicolumn{3}{|c|}{ Implant Type } \\
\hline $\begin{array}{l}\text { multiple } \\
\text { m Standardized Ring }\end{array}$ & $\begin{array}{l}\text { 3D Printed with } \\
\text { Linear Channels }\end{array}$ & $\begin{array}{l}\text { 3D Printed with } \\
\text { Curved Channels }\end{array}$ \\
\hline 1 & 20.49 & 14.58 & 14.46 \\
\hline 5 & 29.11 & 25.24 & 16.18 \\
\hline 10 & 35.04 & 29.73 & 19.52 \\
\hline 15 & 41.51 & 31.97 & 22.87 \\
\hline
\end{tabular}

TABLE I

THE MINIMUM COVERAGE RADIUS $\delta$ (IN MM) NEEDED TO ACHIEVE $100 \%$ COVERAGE $\mathcal{Q}$, FOR $1,5,10$, AND 15 DWELL POINTS RESPECTIVEL. THE 3D PRINTED IMPLANT WITH CURVED CHANNELS, RIGHTMOST COLUMN, ACHIEVES FULL COVERAGE WITH SMALLER COVERAGE RADIUS IN ALL CASES. 

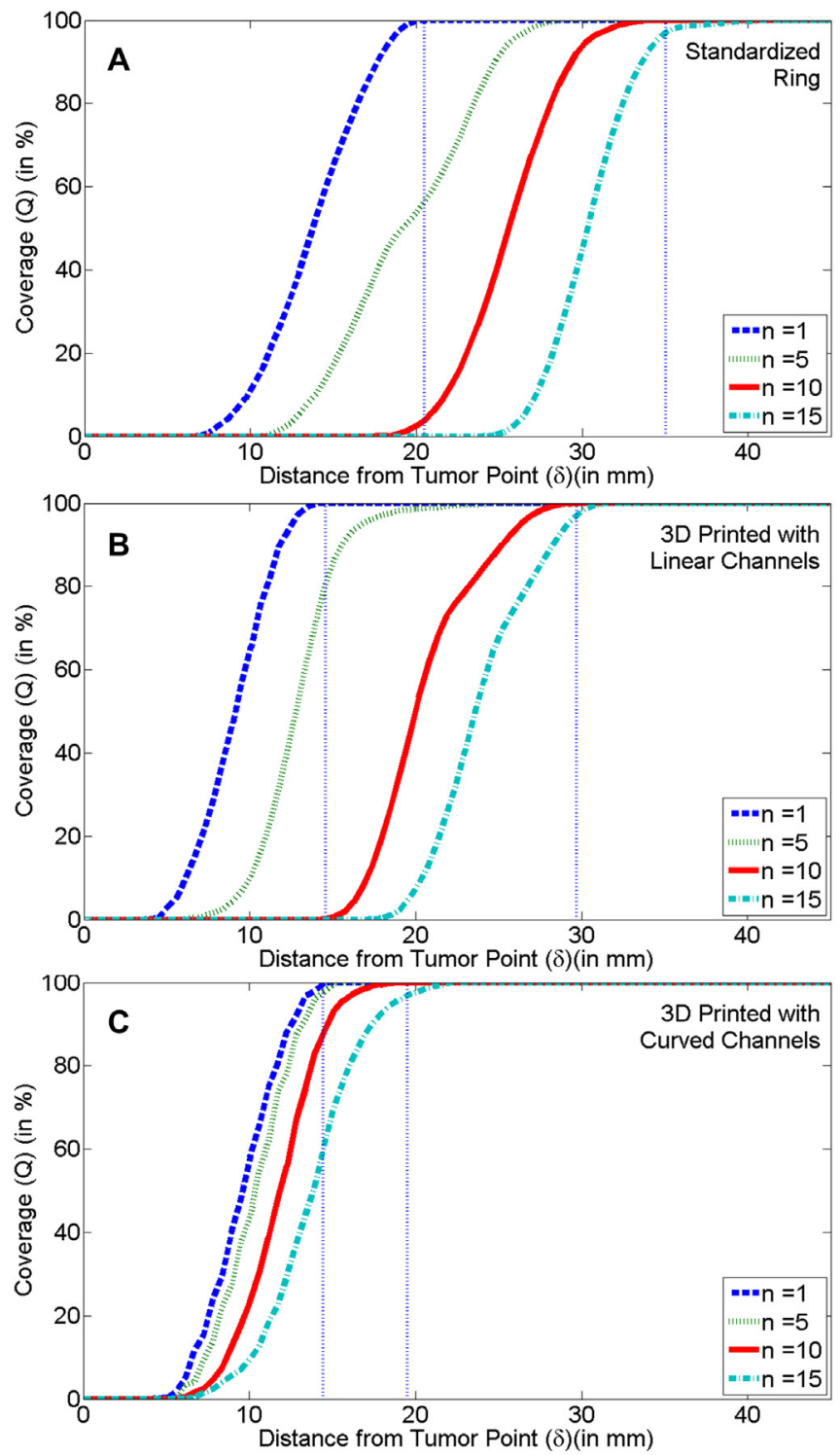

Fig. 8. Coverage metric for each of three treatment options: standardized ring implant (current practice), customized 3D printed implant with linear channels, and customized 3D printed implant with curved channels. Plot of quality $\mathcal{Q}$ (percentage of tumor volume covered) at radiation radius of $\delta$ for $1,5,10$, and 15 dwell positions respectively. (A) standardized ring implant. (B) 3D Printed implant with linear channels, and (C) 3D Printed implant with curved channels. The dashed vertical lines in each plot indicate the value of $\delta$ at which $\mathcal{Q}=100 \%$ is achieved for $n=1$ and $n=10$ respectively. Full tumor coverage is achieved with significantly lower radii in case (C).

practice. Such improvements in the coverage metric increase options for dose planning, which can reduce occurrence of hot spots and increase dose conformality to the tumor geometry to spare healthy tissue.

We envision that such 3D printed implants are clinically viable as outlined in the following potential treatment workflow.

1) Create Plaster Cast: Following the clinical approach described in Magne et al. [3]. Alternatively perform a pre-implant patient scan with CT or MRI and subsequently perform image co-registration.

2) 3D Scan Plaster Cast: Scan 3D geometry of plaster cast exterior, noting locations of tumors on boundary when

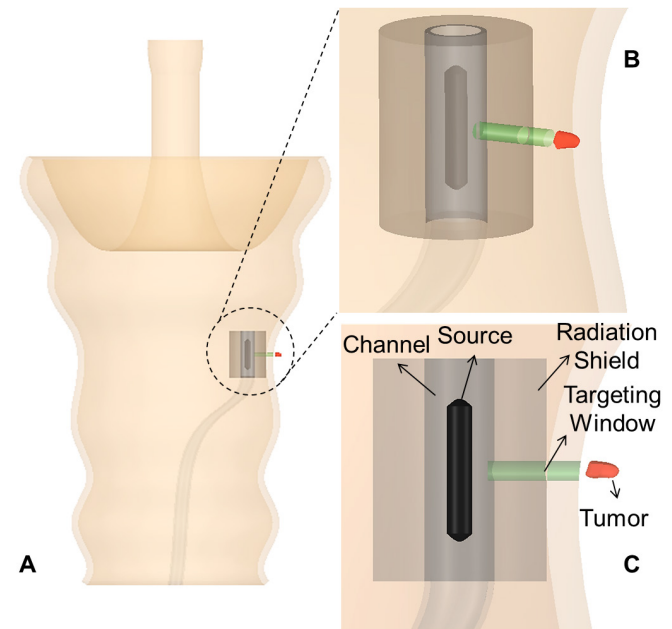

Fig. 9. Conceptual illustration of how lead shielding could be incorporated into the implant as it is now possible to include multiple materials during 3D printing fabrication. (A) illustrates a channel proximal to a small tumor shown in red. $(\mathrm{B}, \mathrm{C})$ are close-up views of the co-axial source, channel, and lead shielding, the latter with a small cylindrical void that serves as a "targeting window" to allow radiation to be emitted toward the tumor while shielding nearby healthy tissue.

possible.

3) 3D Print Planning Implant with Registration Fiducials: Print implant with embedded CT and/or MRI readable fiducial markers.

4) CT or MRI Scan of patient with inserted Planning Implant: The patient is scanned using CT and/or MRI. This generates a 3D model of specific patient anatomy with associated fiducial marks embedded in the implant. On 3D model, clinician identifies the accurate size, shape and position of tumors and also the healthy organs in vicinity.

5) Compute Dose Plan and Channels: Use Channel Layout Algorithm (CLA) with Inverse Dose Planning software to compute a set of channels and dwell points to achieve dose objectives and minimize damage to healthy tissue.

6) 3D Print Resulting Implant with Internal Channels

7) Insert 3D Printed Implant and apply treatment: Radiation source is moved through channels by programmed afterloader over several sessions as needed.

In future work, we will explore a number of extensions and study how the concept can be applied to other anatomical intracavity locations. We are now working on an extended case study where we will 3D print a customized model and incorporate the IPIP [32] inverse dose planning algorithm with the CLA algorithm to generate channels and dwell times. For selecting the set of dwell segments, we will implement the efficient approximation algorithm proposed by Arkin et al. [29] for the "Pencil Packing Problem". We will also consider how a single channel might reach several desired dwell segments and alternate heuristics for ordering dwell segments.

There are also several exciting extensions that can be facilitated by innovations in 3D printing. For example, emerging 
3D printing technologies allow printing of a wide variety of materials including FDA-approved plastics, resins, and composites. Multiple materials can be printed in sequence, allowing complex devices to be printed. Metals such as aluminum, silver, and lead can also be printed, so it may also be possible to incorporate lead shielding into the implant as it is fabricated. This has potential to shield healthy tissue and direct radiation to small tumor targets as illustrated in Figure 9.

\section{ACKNOWLEDGMENT}

This research was funded in part by NSF Awards IIS1227406 and ARRA 0905344. We thank Zach Mulder for his help finding the model of human anatomy for the case study and the staff at UCSF Medical Center at Mt. Zion, San Francisco, CA for input on the clinical procedures for GYN HDR brachytherapy. We would also like to thank Ben Kehoe for offering valuable advice in improving the paper.

\section{REFERENCES}

[1] J. Valentin et al., "Prevention of High-dose-rate Brachytherapy Accidents. ICRP Publication 97.," Annals of the ICRP, vol. 35, no. 2, p. 1, 2005.

[2] K. Wallner, J. C. Blasko, and M. Datolli, Prostate Brachytherapy Made Complicated. Seattle, WA: Smart Medicine Press, 2001.

[3] N. Magné, C. Chargari, N. SanFilippo, T. Messai, A. Gerbaulet, and C. Haie-Meder, "Technical aspects and perspectives of the vaginal mold applicator for brachytherapy of gynecologic malignancies," Brachytherapy, vol. 9, no. 3, pp. 274-277, 2010.

[4] J. Borg and D. Rogers, "Monte carlo calculations of photon spectra in air from 192ir sources," National Research Council Report PIRS-629r, Ontario, Canada, 1999.

[5] Y. Y. Huang and K. H. Low, "Comprehensive planning of robotic therapy and assessment of task-oriented functions via improved $\{\mathrm{QFD}\}$ applicable to hand rehabilitation," in Automation Science and Engineering (CASE), 2010 IEEE Conference on, pp. 252-257, IEEE, 2010.

[6] K. Tervo, L. Palmroth, and H. Koivo, "Skill Evaluation of Human Operators in Partly Automated Mobile Working Machines," IEEE Transactions on Automation Science and Engineering, vol. 7, pp. 133142, Jan. 2010.

[7] J. Solis and A. Takanishi, "Towards enhancing the understanding of human motor learning," in 2009 IEEE International Conference on Automation Science and Engineering, pp. 591-596, IEEE, Aug. 2009.

[8] J. A. Mendez, S. Torres, J. A. Reboso, and H. Reboso, "Model-based controller for anesthesia automation," in 2009 IEEE International Conference on Automation Science and Engineering, pp. 379-384, IEEE, Aug. 2009.

[9] K. Subburaj, B. Ravi, and M. G. Agarwal, "Automated 3D geometric reasoning in Computer Assisted joint reconstructive surgery," in 2009 IEEE International Conference on Automation Science and Engineering, pp. 367-372, IEEE, Aug. 2009.

[10] A. Garg, T. Siauw, D. Berenson, A. Cunha, I.-C. Hsu, J. Pouliot, D. Stoianovici, and K. Goldberg, "Initial experiments toward automated robotic implantation of skew-line needle arrangements for \{HDR $\}$ brachytherapy," in Automation Science and Engineering (CASE), 2012 IEEE International Conference on, pp. 26-33, 2012.

[11] R. Potter and C. Kirisits, "Upcoming ICRU/GEC ESTRO recommendations for brachytherapy in cancer of the Cervix (1)," Radiotherapy and Oncology, vol. 103, p. S42, 2012.

[12] R. Pötter, C. Haie-Meder, E. V. Limbergen, I. Barillot, M. D. Brabandere, J. Dimopoulos, I. Dumas, B. Erickson, S. Lang, A. Nulens, and Others, "Recommendations from gynaecological (GYN) GEC ESTRO working group (II): Concepts and terms in 3D image-based treatment planning in cervix cancer brachytherap 3D dose volume parameters and," Radiotherapy and oncology, vol. 78, no. 1, pp. 67-77, 2006.
[13] L. Delclos, G. H. Fletcher, E. Bailey Moore, and V. A. Sampiere, "Minicolpostats, dome cylinders, other additions and improvements of the Fletcher-Suit afterloadable system: Indications and limitations of their use," International Journal of Radiation Oncology* Biology* Physics, vol. 6, no. 9, pp. 1195-1206, 1980.

[14] M. Bernstein, K. J. Mehta, R. Yaparpalvi, H. Kuo, and S. Kalnicki, "Results of the Hybrid Interstitial-Intracavitary Utrecht Aapplicator for cervical cancer in an Outpatient setting," Radiotherapy and Oncology, vol. 103, p. S116, 2012.

[15] J. C. A. Dimopoulos, C. Kirisits, P. Petric, P. Georg, S. Lang, D. Berger, and R. Pötter, "The Vienna applicator for combined intracavitary and interstitial brachytherapy of cervical cancer: Clinical feasibility and preliminary results," International Journal of Radiation Oncology*Biology*Physics, vol. 66, no. 1, pp. 83-90, 2006.

[16] J. N. Roy, K. E. Wallner, L. L. Anderson, and C. Ling, "CTbased optimized planning for transperineal prostate implant with customized template," International Journal of Radiation Oncology*Biology*Physics, vol. 21, pp. 483-489, July 1991.

[17] H. Lipson and M. Kurman, Fabricated: The New World of 3D Printing. Wiley, 2013.

[18] N. Gershenfeld, "Fab: The coming revolution on your desktop-from personal computers to personal fabrication," 2007.

[19] P. F. Jacobs and D. T. Reid, Rapid prototyping \& manufacturing: Fundamentals of stereolithography. Society of Manufacturing Engineers in cooperation with the Computer and Automated Systems Association of SME, 1992.

[20] F. P. W. Melchels, J. Feijen, and D. W. Grijpma, "A review on stereolithography and its applications in biomedical engineering.," Biomaterials, vol. 31, pp. 6121-30, Aug. 2010.

[21] H. Seitz, W. Rieder, S. Irsen, B. Leukers, and C. Tille, "Threedimensional printing of porous ceramic scaffolds for bone tissue engineering.," Journal of biomedical materials research. Part B, Applied biomaterials, vol. 74, pp. 782-8, Aug. 2005.

[22] J. D'haese, T. Van De Velde, A. Komiyama, M. Hultin, and H. De Bruyn, "Accuracy and complications using computer-designed stereolithographic surgical guides for oral rehabilitation by means of dental implants: a review of the literature.," Clinical implant dentistry and related research, vol. 14, pp. 321-35, June 2012.

[23] V. Duindam, R. Alterovitz, and K. Goldberg, "Motion planning for steerable needles in $3 \mathrm{D}$ environments with obstacles using rapidlyexploring Random Trees and backchaining," in 2008 IEEE International Conference on Automation Science and Engineering, pp. 41-46, IEEE, Aug. 2008.

[24] S. Patil and R. Alterovitz, "Interactive Motion Planning for Steerable Needles in 3D Environments with Obstacles.," Proceedings of the ... IEEE/RAS-EMBS International Conference on Biomedical Robotics and Biomechatronics. IEEE/RAS-EMBS International Conference on Biomedical Robotics and Biomechatronics, pp. 893-899, Jan. 2010.

[25] N. J. Cowan, K. Goldberg, G. S. Chirikjian, G. Fichtinger, R. Alterovitz, K. B. Reed, V. Kallem, W. Park, S. Misra, and A. M. Okamura, "Robotic needle steering: Design, modeling, planning, and image guidance," in Surgical Robotics: System Applications and Visions (J. Rosen, B. Hannaford, and R. M. Satava, eds.), ch. 23, pp. 557-582, Springer, 2011.

[26] M. Hwangbo, J. Kuffner, and T. Kanade, "Efficient Two-phase 3D Motion Planning for Small Fixed-wing UAVs," in Proceedings 2007 IEEE International Conference on Robotics and Automation, pp. 1035-1041, IEEE, Apr. 2007.

[27] J. Le Ny, E. Feron, and E. Frazzoli, "On the Dubins Traveling Salesman Problem," IEEE Transactions on Automatic Control, vol. 57, pp. 265-270, Jan. 2012.

[28] S. M. LaValle, Planning Algorithms. Cambridge, U.K.: Cambridge University Press, 2006. Available at http://planning.cs.uiuc.edu.

[29] E. M. Arkin, S. P. Fekete, J. Kim, J. S. Mitchell, G. R. Sabhnani, and J. Zou, "The pencil packing problem," 2009.

[30] G. van den Bergen, Collision detection in interactive 3D environments. Morgan Kaufmann, 2004.

[31] K. T. Barnhart, A. Izquierdo, E. S. Pretorius, D. M. Shera, M. Shabbout, and A. Shaunik, "Baseline dimensions of the human vagina.," Human reproduction (Oxford, England), vol. 21, pp. 1618-22, June 2006.

[32] T. Siauw, A. Cunha, A. Atamtürk, I.-C. Hsu, J. Pouliot, and K. Goldberg, "Ipip: A new approach to inverse planning for hdr brachytherapy by directly optimizing dosimetric indices," Medical Physics, vol. 38, p. 4045, 2011. 